\title{
Métodos e técnicas de gestão do conhecimento aplicadas para melhorar a gestão do capital intelectual em núcleos setoriais de uma associação empresarial
}

\section{RESUMO}

A importância do conhecimento, demonstrada ao longo dos séculos, é fundamental para o desenvolvimento ambiental, econômico e social de qualquer organização. A melhoria da identificação, criação, estoque, disseminação e aplicação do conhecimento é forma de desenvolver e aprimorar os processos organizacionais no sentido do desenvolvimento sustentável, no qual o Capital Intelectual é fundamental. Há vários trabalhos que demonstram melhorias nos resultados de empresa que gestionam seus capitais humano, estrutural e relacional. Os objetivos principais da pesquisa foram caracterizar e analisar as técnicas e os métodos de Gestão do Conhecimento (GC) usados por empresas de dois núcleos setoriais da Associação Comercial e Industrial de Jaraguá do Sul (ACIJS). A amostra incluiu dez empresas, correspondendo a $25 \%$ dos participantes ativos dos núcleos setoriais: Núcleo de Sustentabilidade Empresarial e Núcleo de Gestão e Qualidade. Foi aplicado um questionário elaborado pela Asian Productive Organization (APO, 2010) aos representantes das empresas dos núcleos setoriais. As respostas caracterizaram a situação atual e a situação desejada, graduadas numa escala de zero a quatro. Constatou-se que existe um conjunto significativo de métodos e técnicas de GC já implementados nas empresas, que, se corretamente utilizados, suportariam uma adequada gestão do seu capital intelectual. Os principais métodos e técnicas aplicados foram ajuda a semelhante, reuniões informais, visitas técnicas entre empresas, viagens e visitas a feiras, elaboração de manuais para replicação de práticas, reembolso de despesas, portais eletrônicos: portais - e-mail - chats. No entanto, somente duas foram avaliadas, como tendo realmente um grande alcance dentro das organizações. Foram elas: reuniões informais e reembolso de despesas. Vinte métodos e técnicas foram elencados como uma situação desejada para grande aplicação nas empresas.

Palavras-chave: Gestão do Conhecimento. Métodos de Gestão do Conhecimento. Técnicas. Capital intelectual. 


\section{INTRODUÇÃO}

As ações de aquisição, armazenamento e compartilhamento de conhecimentos, de modo a aprender as lições do passado e de outros lugares - a superação dos limites impostos pelo tempo e pelo espaço - estão longe de serem novidades. Nos últimos anos, atuações nesse sentido enfatizaram a melhor aplicação do conhecimento e da aprendizagem como um meio de melhorar o trabalho e o desenvolvimento humanitário.

Durante a Era Industrial, qualquer empresa, não importa quão grande ou pequena fosse ou o setor ao qual pertencesse, poderia crescer e prosseguir com melhorias na produtividade e qualidade, se fizesse as "coisas direito". As empresas concorreram para a eficiência, e o esquema competitivo foi bastante estático. No entanto, segundo a Asian Productivity Organization (APO), desde o início de 1990, o paradigma da competição mudou gradualmente, "da guerra da eficiência para a guerra para a inovação como o campo de batalha" (APO, 2009, p. 01). E, nesse aspecto, Drucker (2001) afirma que, na sociedade do conhecimento, se vivem mudanças de paradigmas, como foco inquestionável de transformação: o conhecimento como fator-chave de riqueza e prosperidade.

Os eventos conduziram a estratégias baseadas em conhecimento e adoção da aprendizagem. Dentre os órgãos envolvidos nesse tipo de tarefa, incluem-se agências financiadoras multilaterais, governos, institutos de pesquisa, organizações regionais, Organizações Não Governamentais (ONGs) e organizações privadas com fins lucrativos.

Assim, os sistemas produtivos contemporâneos, nas próximas décadas, deverão adaptarse aos mercados globalizados e direcionar seus ativos intangíveis de conhecimentos ao direito, à propriedade intelectual, à pressão crescente da sociedade exigente em serviços e bens sadios e em quantidade, oriundos de modelos que preconizem o desenvolvimento sustentável.

Tanto o conhecimento e a gestão do conhecimento (GC) são complexos de definir. Acadêmicos no campo da GC normalmente definem conhecimento como um derivado de informação, que é proveniente a partir dos dados. Enquanto informações são dados organizados com um significado (NORTH; RIVAS, 2010), o conhecimento é informação contextualizada que possibilita agir (DRUCKER, 1999). A ideia central é que os esforços de GC devam trabalhar para criar, codificar, compartilhar e armazenar conhecimentos que permitam a criação de valor na organização. Segundo APO (2009), a criação de valor acontece em duas dimensões: Desempenho e Aprendizagem. Outros pensamentos indicam que a GC muda o foco do processo de prática. A GC deve ser usada para comunicação e a colaboração para aprimorar a forma como as pessoas fazem a sua prática (o seu trabalho dentro de um processo global) (GHANI, 2009; HEISIG, 2009; PORTER; KRAMMER, 2011; SENGE, 2000). APO (2009) esclarece que, se o conhecimento é o principal recurso produtivo na atualidade, sua gestão é totalmente prioritária nas empresas de diversas dimensões. Dessa forma, torna-se um desafio nas várias organizações, pois a gestão adequada do conhecimento, como elemento agregador de valor, deve estar alinhada e direcionada com a própria operação, para um uso ótimo dos recursos.

A competência de gerir o conhecimento organizacional diz respeito à forma de uma organização olhar para o seu conhecimento e aprendizagem de forma estratégica. Os métodos de GC fornecem diferentes aplicações no capital humano, estrutural e relacional que podem ser usados para planejar, monitorar e avaliar o conhecimento e iniciativas de aprendizagem (APO, 2009; RAMALINGAM, 2006). De acordo com Idea (2008), os métodos de GC englobam o uso de ideias e experiências dos funcionários, clientes e fornecedores, e a estrutura para melhorar o desempenho da organização, com base no que funciona bem, leva a uma melhor prática, estratégia e política.

Os métodos e técnicas de GC proporcionam o entendimento das abordagens dos métodos e técnicas usados frequentemente na GC nas organizações. Em particular, os métodos fornecem passos essenciais para mapear a GC como instrumento para identificar, criar, disseminar, estocar e aplicar conhecimento nas organizações, no sentido de gerar sustentabilidade nestas (APO, 2010; HEISIG, 2009; CEN, 2004). De certo modo, os métodos são destinados aos usuários com compreensão, em profundidade, nos fundamentos sobre GC.

Nesse sentido, a APO (2010) apresenta um manual a respeito do tema, desenvolvido por uma equipe de especialistas asiáticos, europeus e norte-americanos. Essa equipe organizou uma lista composta de metas e etapas para, em cinco fases, mapear a GC nas organizações, uma maneira prática, para ganhos dentro da organização. Os métodos de GC foram desenvolvidos com a finalidade de apoiar os processos-chave de projetos e negócios de uma organização. Assim, observa-se a importância de obter equilíbrio entre os processos de criação, disseminação, ação dos empreendimentos e dos métodos de GC.

O presente estudo proporciona um arrolamento do uso de métodos de GC, com base no 
método APO em um cluster do conhecimento, sob determinantes da gestão do conhecimento para desenvolvimento local sustentável, em que o indivíduo, as organizações e a sociedade possuem papel preponderante para o desenvolvimento do Capital Intelectual. Os objetivos foram caracterizar e avaliar os métodos e técnicas de GC usados pelas empresas de dois núcleos setoriais da Associação Comercial e Industrial de Jaraguá do Sul (ACIJS), com participantes ativos dos núcleos setoriais Núcleo de Sustentabilidade Empresarial e Núcleo de Gestão e Qualidade. Fundada em 1938, a ACIJS tem como missão o estímulo ao empreendedorismo com responsabilidade social. Por meio de programas de capacitação, benefícios e soluções empresariais, a entidade exerce representatividade junto ao poder público e tem uma ativa participação comunitária em favor do município e da região.

\section{DESENVOLVIMENTO}

A seguir são apresentadas e analisadas as pesquisas que sustentam o presente trabalho, assim como os constructos utilizados para analisar de que forma as práticas de GC podem melhorar a gestão do $\mathrm{Cl}$, a fim de agregar valor às organizações.

\subsection{Métodos e técnicas de GC}

Os elementos condutores e facilitadores para a implementação de projeto de GC, de acordo com Dalkir (2005), são liderança, pessoas, processos e tecnologias. Os líderes conduzem as iniciativas e garantem o alinhamento estratégico, apoio e recursos na organização. Pessoas são importantes, pois são o elemento central da gestão do conhecimento, principalmente no tocante à criação, aplicação e compartilhamento de conhecimento, e formam um repositório ativo de conhecimento tácito (e até mesmo conhecimento explícito até que possa ser documentado). As pessoas são parte do capital humano, elas criam e possuem capital intelectual. O sucesso dos projetos de GC, em grande parte, depende da disposição das pessoas em compartilhar conhecimento, referenda Young (2010). Processos são eventos que descrevem o funcionamento na organização e podem contribuir para melhorar o desempenho da organização. As tecnologias aceleram os processos de GC, fornecendo técnicas e métodos, tais como motores de busca, mídias de armazenamento, intranets e extranets.

Dalkir (2005) apresenta um conjunto de modelos, processos, ciclos e, por fim, métodos e técnicas utilizados para gerenciar o conhecimento. Os métodos e técnicas são agrupados dentro dos processos de criação, identificação, aplicação, armazenamento e compartilhamento do conhecimento. $O$ mesmo autor esclarece que os métodos podem ser combinados trabalhando juntos para alcancar objetivos de negócio. Nessa mesma linha, Servin (2005) explica que uma boa estratégia de GC deve responder a três questões-chave: Onde estamos? Aonde queremos ir? Como chegaremos lá?

O Guia Europeu de Melhores Práticas de GC, publicado pelo Comité Europeén de Normatisation (CEN, 2004) aponta as pessoas, os processos e as tecnologias como os fatores essenciais que determinam o sucesso ou o fracasso de uma iniciativa de gestão do conhecimento nas organizações. Esses elementos e suas relações constituem o quadro de referência do CEN (2004). Normalmente, um quadro de referência é concebido com base em uma representação gráfica que mostra como implantar a GC em uma organização. Vários quadros de referências estão hoje disponíveis na literatura: Australian KM Standard, APQC, CEN, APO, etc.

APO (2010) desenvolveu um quadro referencial para GC (Figura 1) para o entendimento dos aspectos estratégicos, operacionais e dos resultados da GC nas organizações. É pertinente salientar que o quadro referencial da APO presupõe que todas as organizações têm uma GC; portanto, o seu foco é desenvolver implementações orientadas a aprimorar essa gestão. Assim, o quadro referencial permite identificar e planejar ações de diagnóstico de projeto, implementação, monitoramento e maturidade de programas de GC nas organizações.

O quadro referencial da APO (2010) apresenta sete dimensões para avaliar a GC numa organização, organizados em três grupos: (1) os quatro aceleradores da GC - pessoas, processos, tecnologia e liderança-, existentes em todas as organizações como elementos que suportam a GC. (2) Os processos de GC propriamente ditos, ou seja, de criação, identificação, aplicação, armazenamento e compartilhamento do conhecimento. (3) Duas dimensões de resultados - de aprendizagem e inovação, e de resultados da GC -, que caracterizam objetivos em termos de aprendizagem e desempenho que toda implementação bem-sucedida de GC deve atingir.

Os processos de GC (criação, identificação, aplicação, armazenamento e compartilhamento) possibilitam a valoração a partir do conhecimento, isto é, colocam o conhecimento em ação efetiva 
orientada a resultados estratégicos (DRUCKER, 1999). Dessa forma, existe um conjunto de práticas de GC que apoiam e suportam a execução de cada um dos processos de GC. O quadro de referência da APO (2010) apresenta tambem essas práticas de GC, as quais podem ser agrupadas como: Questões de troca de conhecimento orientadas ao compartilhamento; Práticas para conexão entre pessoas; Memória organizacional; e Mecanismos de coordenação.

Figura 1 - Quadro Referencial de Gestão do Conhecimento

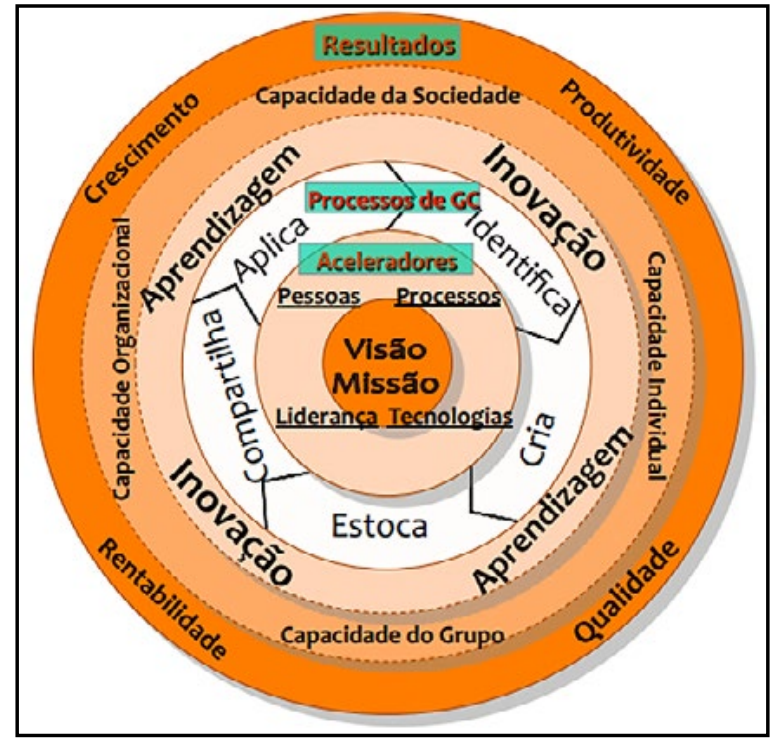

Fonte: Adaptado de APO (2010, p. 28)

Em decorrência dos seus objetivos (caracterizar e analisar os métodos e técnicas de GC na prática), a presente pesquisa utiliza as práticas de GC apresentadas pela APO (2010) como elementos-chave para suportar os processos de GC. Tais práticas permitem utilizar as dimensões aceleradoras, atingir os objetivos de GC nas dimensões de aprendizagem e inovação e de resultados de desempenho.

\subsection{Capital intelectual na perspectiva do capital social como mecanismo de desenvolvimento} sustentável

A relação entre $\mathrm{Cl}$ e GC é importante, porque os relatórios do capital intelectual informam sobre as atividades que o gerenciamento inicia e apoia em nome da gestão do conhecimento (BUKH; LARSEN; MOURITSEN, 2001).

$\mathrm{Na}$ economia do conhecimento de hoje, a gestão tradicional de ativos e passivos financeiros não pode mais garantir vantagem competitiva. A diferença entre o valor de mercado (a avaliação do mercado de capitais sobre o futuro potencial de desenvolvimento da empresa) e o valor contábil (o atual valor contábil do investimento passado calculado por um método de contabilidade) mostram a importância do CI (TSENG et al., 2013; BONTIS, 2001; BUENO; SALMADOR; RODRÍGUEZ, 2006).

De acordo com Nahapiet e Ghoshal (1998), o capital intelectual de uma organização não é propriedade de um indivíduo, mas resulta e desenvolve-se nas relações que permeiam a estrutura e os processos de troca, é moldado de acordo com a evolução dessas relações ao longo do tempo. É, portanto, de difícil imitação e raro, pois é característico de cada organização; deve ser valioso, gerar produtos capazes de proporcionar atratividade durável ao cliente, superior à da concorrência.

O conceito de $\mathrm{Cl}$ expande e aprofunda a compreensão estratégica e operacional de capacidadeschave das empresas. Na última década, o referido conceito emergente estabeleceu-se entre as construções de gestão mais amplamente aceitas. Sua abordagem holística enfatiza as dimensões do conhecimento empregado e ativado nas organizações, bem como o impacto dos recursos baseados no conhecimento sobre o seu desempenho. Seu principal objetivo é fornecer às empresas orientações sobre como desenvolver suas principais capacidades representadas no capital intelectual, medir a sua contribuição e gerir o seu crescimento, e, mais importante, encontrar a melhor e mais produtiva forma de criar um valor ao considerar a especificidade de suas capacidades-chave e efeitos que surgem a partir de suas interações (KOMNENIC; POKRAJCIC, 2012).

Os diferentes elementos do Capital Intelectual são constituídos por contribuiç̧ões de vários autores com diferentes teorias e práticas. Conforme Rodriguez e Stoeckicht (2004), o Cl converge em 
três categorias: As questões relacionadas às pessoas - habilidades, educação, experiência, valores, motivações, etc. Os aspectos internos - organização, forma de organização, tecnologia, patentes, conceitos, computadores, cultura, administração empresarial, etc. E, por fim, as considerações para o estabelecimento de relações com o exterior da empresa - clientes, fornecedores, acionistas, instituições, etc.

De acordo com Giuliani e Marasca (2011), o primeiro passo de qualquer avaliação é a definição do objeto a ser valorizado. Valorar $\mathrm{Cl}$ é defini-lo, ou melhor, determinar seus limites. Limites, na verdade, são essenciais, a fim de distinguir uma parte do conjunto e, por conseguinte, determinar o seu valor para além do valor da empresa. Ao mesmo tempo, os limites podem ser considerados estruturas de ligação, isto é, itens que produzem e reproduzem a unidade interna de uma entidade. Neste estudo, o termo "definição" será utilizado para fazer referência à escolha da definição teórica adotada, enquanto o termo "identificação" será utilizado para descrever o desenho dos limites do $\mathrm{Cl}$.

De acordo com Calvo et al. (2002), três categorias são definidas no relatório de Cl. O capital humano é definido como o conhecimento que os funcionários levam consigo quando saem da empresa. Referido capital inclui os conhecimentos, habilidades, experiências e habilidades das pessoas. Parte desse conhecimento é exclusivo para o indivíduo, alguns podem ser genéricos. $\mathrm{O}$ capital estrutural é definido como o conhecimento que permanece dentro da empresa, no fim do dia de trabalho. Compreende as rotinas organizacionais, procedimentos, sistemas, culturas, bases de dados, etc. O capital relacional é definido como todos os recursos vinculados às relações externas da empresa com clientes, fornecedores ou parceiros de P\&D. Compreende parte do capital humano e estrutural envolvido com as relações da empresa com as partes interessadas (investidores, credores, clientes, fornecedores, etc), além das percepções que tais interessados têm sobre a empresa. Essa estruturação em Capital Humano, Capital Estrutural e Capital Relacional foi referendada pelo estudo de Ferenhof, Bialecki e Selig (2013). No Quadro 1, estão distribuídos os métodos e técnicas de GC, quanto as categorias do $\mathrm{Cl}$.

Quadro 1 - Contribuição das práticas de GC na gestão do Capital Intelectual

\begin{tabular}{|c|c|c|}
\hline \multicolumn{3}{|c|}{ CAPITAL INTELECTUAL } \\
\hline CAPITAL HUMANO & CAPITAL ESTRUTURAL & CAPITAL RELACIONAL \\
\hline \multicolumn{3}{|c|}{ Conexões interpessoais } \\
\hline 1 - Comunidade de prática & & 1 - Feiras do conhecimento \\
\hline \multicolumn{3}{|l|}{2 - Café do conhecimento } \\
\hline \multicolumn{3}{|l|}{3 - Ajuda Semelhante } \\
\hline \multicolumn{3}{|c|}{ Memória Organizacional } \\
\hline 4 - Revisão pós-ação & 1 - Foi certo ou foi errado & 2 - Fóruns presenciais e virtuais \\
\hline 5 - Revisão retrospectiva & 2 - Publicações técnicas & \\
\hline \multicolumn{3}{|l|}{6 - Troca de conhecimento } \\
\hline \multicolumn{3}{|l|}{7 - Reuniões informais } \\
\hline \multicolumn{3}{|l|}{8 - Atividades conjuntas } \\
\hline \multicolumn{3}{|l|}{9 - Grupos para solução } \\
\hline \multicolumn{3}{|c|}{ Mecanismo de Coordenação Social - Controle e Coordenação } \\
\hline 10 - Mestre aprendiz & 3 - Espaço físico compartilhado & 3 - Visitas técnicas \\
\hline 11 - Coaching & & 4 - Observação real \\
\hline \multicolumn{3}{|l|}{12 - Conselheiro de campo } \\
\hline \multicolumn{3}{|c|}{ Mecanismo de Coordenação Social - Controle e Coordenação } \\
\hline 13 - Confraternização & & 5 - Viagens, visitas a feiras \\
\hline \multirow[t]{2}{*}{14 - História compartilhada } & & 6 - Congressos \\
\hline & & $\begin{array}{l}7 \text { - Ações de responsabilidade social e } \\
\text { ambiental }\end{array}$ \\
\hline \multicolumn{3}{|c|}{ Mecanismo de Coordenação Social - Controle e Planejamento } \\
\hline 15 - Narrativas & 4 - Compartilhamento de melhores práticas & \\
\hline & $\begin{array}{l}5 \text { - Elaboração de manuais para replicar } \\
\text { boas práticas }\end{array}$ & \\
\hline
\end{tabular}




\begin{tabular}{|c|c|c|}
\hline & 6 - Rotinas bem-sucedidas & \\
\hline & 7 - Memória organizacional & \\
\hline & 8 - Sistema de inteligência competitiva & \\
\hline & 9. Benchmarking interno e externo & \\
\hline \multicolumn{3}{|c|}{ Sistema de Incentivo } \\
\hline 16 - Estimulo à inovação & 10 - Dia de inovação & \\
\hline \multicolumn{3}{|l|}{$\begin{array}{l}\text { 17- Treinamentos, cursos, } \\
\text { palestras e workshop }\end{array}$} \\
\hline \multicolumn{3}{|l|}{18 - Educação continuada } \\
\hline \multicolumn{3}{|l|}{$19-$ Reembolso de despesas } \\
\hline \multicolumn{3}{|c|}{ Sistema de Informação } \\
\hline & 11 - Espaço eletrônico, e-learning & \\
\hline & 12 - Base de dados compartilhados on- line & \\
\hline & 13 - Gestão de conteúdos & \\
\hline & 14 - Gestão eletrônica de documento & \\
\hline
\end{tabular}

Fonte: Os autores (2013)

\section{METODOLOGIA}

Os objetivos do artigo foram caracterizar e analisar os métodos e técnicas de GC usados pelas empresas de dois núcleos setoriais da ACIJS. A amostra incluiu dez empresas, correspondendo a $25 \%$ dos participantes ativos dos núcleos setoriais Núcleo de Sustentabilidade Empresarial e Núcleo de Gestão e Qualidade. A amostra continha empresas do setor de alimentos, saúde, metal-mecânica, têxtil e serviços.

Preliminarmente, foram realizados dois encontros presenciais, durante as reuniões ordinárias dos dois núcleos setoriais, com a finalidade de explicar os objetivos da pesquisa e o método aplicado à pesquisa. Os questionários foram enviados por correio eletrônico, no período entre junho e agosto de 2013, elaborados com base no modelo APO (2010). Questionaram-se as atividades para conexão entre pessoas, memória organizacional e mecanismos de coordenação (controle e coordenação social, sistemas de controle e planejamento, sistema de incentivo e sistemas de informação). Os respondentes avaliaram os métodos e técnicas utilizados, em uma escala de 0 a 4, para a situação atual e situação desejada, conforma apresenta o Quadro 2. Nas situações de desconhecimento da ferramenta ou técnica, o respondente deveria colocar índice zero para situação atual e indicar o fato.

Quadro 2 - Escala de avaliação e situação aplicadas ao questionário

\begin{tabular}{|c|c|c|c|}
\hline & Situação Atual (como realmente é) & & Situação desejada (como tem que ser) \\
\hline 0 & Não é utilizada em nada & 0 & Não é utilizada em nada \\
\hline 1 & Tem pouco alcance & 1 & Tem pouca aplicação \\
\hline 2 & Certo alcance & 2 & Certa aplicação \\
\hline 3 & Um alcance grande & 3 & Uma aplicação grande \\
\hline 4 & Um alcance muito grande & 4 & Uma aplicação muito grande \\
\hline
\end{tabular}

Fonte: APO (2010)

\section{RESULTADOS}

As atividades relacionadas às conexões de pessoas 'ajuda a semelhantes' foi a técnica mais utilizada, bem como referendada como sendo de "uma aplicação muito grande". A segunda ferramenta mais aplicada foi 'comunidade prática'; porém, não diferiu em valores médios do 'café do conhecimento'. No entanto, 'café do conhecimento' obteve $30 \%$ de frequência "zero", no sentido de desconhecimento da técnica, mesmo valor obtido pela ferramenta 'feira do conhecimento'. Apesar 
da média de 1,2, de menor valor para o grupo, 'feira do conhecimento' obteve como moda a escala "2", o que representa "certo alcance". Para a situação desejada, seguiu a lógica de sua aplicabilidade, foram mais valorados os métodos 'ajuda semelhante' e 'comunidade prática', com escalas de 3,05 (aplicação muito grande) e 2,9, respectivamente. Os resultados podem ser observados na Figura 1.

Conforme Quadro 1, apresenta que a gestão do capital humano da organização torna-se favorecida em virtude da aplicação da prática 'ajuda a semelhantes', a qual permite reduzir curvas de aprendizagem.

Figura 2 - Atividade para Conexão de Pessoas para os núcleos setoriais da ACIJS

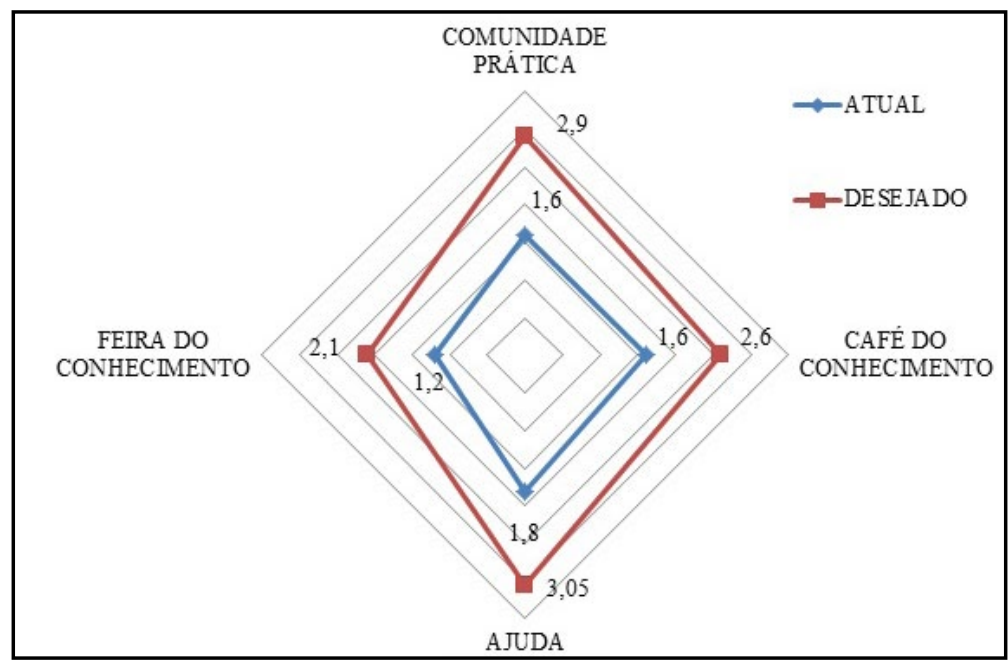

Fonte: Os autores (2013)

Os índices vinculados aos métodos e técnicas relacionados à melhoria organizacional estão presentes na Figura 3. Os valores mais altos para a situação atual foram para as 'reuniões informais'e 'grupos para solução', que obtiveram índice 2.5 e 2.2, respectivamente. Para melhoria organizacional, a técnica 'comunidade prática' obteve os menores índices para utilização $(1,1)$ por resultar em "pouco alcance" e para a situação desejada $(1,9)$.

No âmbito das práticas da melhoria organizacional, a intensa aplicação de reuniões informais e os grupos para solução contribuem para o desenvolvimento das capacidades dos colaboradores e, consequentemente, favorecem a gestão do capital humano da empresa, conforme apresentado no Quadro 1.

Figura 3 - Métodos e técnicas avaliadas para a Melhoria Organizacional para os núcleos setoriais da ACIJS

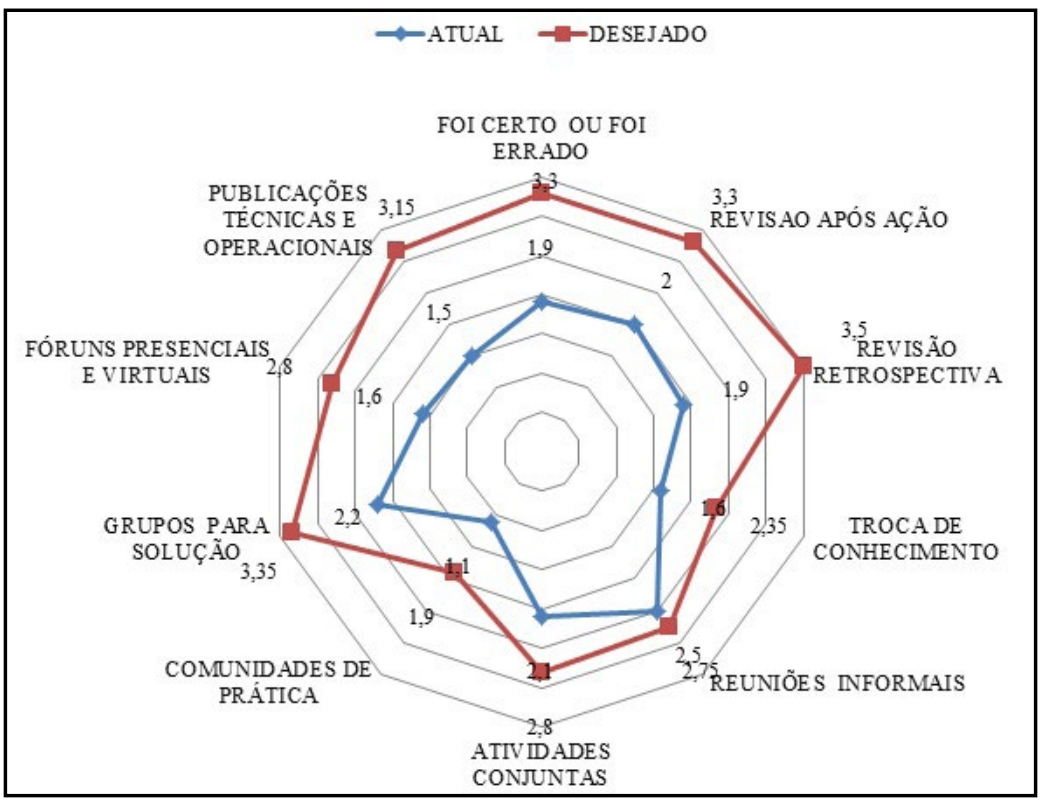

Fonte: Os autores (2013) 
Os métodos de maior importância sendo considerados como "uma aplicação muito grande" (escore superior a três) foram em ordem decrescente de importância 'revisão pós- ação', com 3.5 pontos; seguido por 'grupo para solução' com 3.35 pontos; "foi certo ou foi errado' e 'revisão retrospectiva', que obtiveram média de 3.3 pontos e 3.15 pontos para 'publicações técnicas e operacionais'. Percebe-se, pelos resultados, uma valoração pela identificação das consequências das rotinas aplicadas. As técnicas que obtiveram o menor intervalo entre a situação atual e a desejada foram 'reuniões informais' (0.25 - 2.5 atual e 2.75 desejada) e 'atividades conjuntas' (0.7 - 2.1 atual e 2.8 desejada). 'Revisão retrospectiva' obteve a maior amplitude entre o praticado e o desejado, na ordem, 1.9 e 3.5 .

No que diz respeito aos Mecanismos de coordenação ligados ao controle e coordenação social (Figura 4), destaca-se que a técnica de 'conselheiro de campo' foi utilizada somente em uma organização e teve um índice de aplicação e importância de igual valor. Para o mesmo item, uma empresa não utilizava em nada, mas considerava ter "uma aplicação muito grande". As demais desconheciam a técnica. A segunda maior frequência de falta de conhecimento a respeito da técnica ocorreu com 'mestre aprendiz' e 'espaço compartilhado', presente na resposta em $60 \%$ dos entrevistados. No entanto, 'mestre aprendiz' teve a maior amplitude entre o aplicado e o desejado.

'Observação real' era aplicado em três empresas, com valores semelhantes para o intervalo entre o real e o desejado. Mas, alcançou a maior frequência de falta de conhecimento da técnica. 'Espaço compartilhado', apesar de ter obtido pouca aplicação, teve considerada sua aplicação ideal entre o realizado e desejado, para $3 / 4$ dos entrevistados.

A ferramenta de maior ocorrência foi 'visita técnica entre empresas' e obteve o maior escore de aplicabilidade, 3.5 pontos. A segunda técnica relacionada foi 'coaching', tanto para aplicação como para situação desejada. Assim sendo, essas duas opções para controle e coordenação social obtiveram as maiores frequências para situação desejada, com escore "4".

O uso das técnicas "visita técnica entre empresas" e "coaching" confirma a boa gestão do capital relacional nas empresas participantes, de fato, uma das fortalezas da ACIJS que a torna referência no país.

Figura 4 - Métodos e técnicas avaliadas para Mecanismos de Coordenação Social para controle e coordenação social para os núcleos setoriais da ACIJS

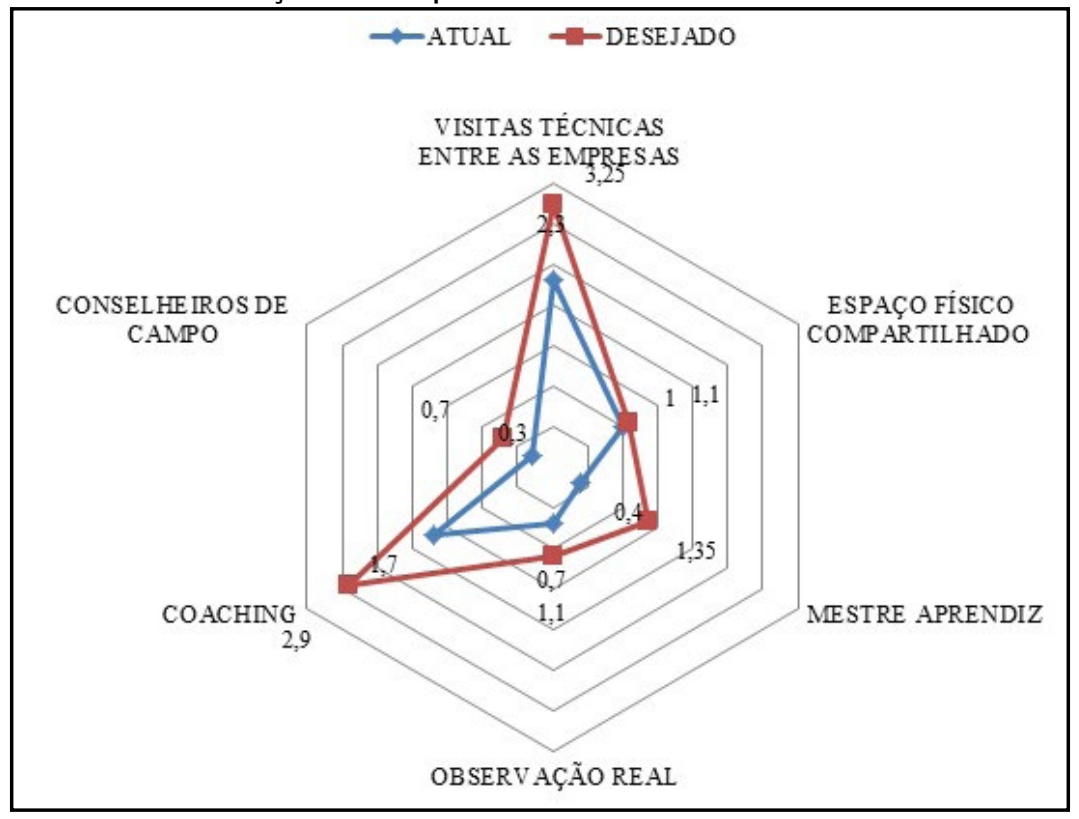

Fonte: Os autores (2013)

A Figura 5 apresenta os resultados para Mecanismos de coordenação relacionados a controle e coordenação social. Constata-se que 'ações de responsabilidade social' obteve o escore de aplicação desejada mais elevado, 3.85, e a segunda ação mais aplicada entre os métodos avaliados (2.7). 0 escore máximo - 'uma aplicação muito grande' - esteve frequente em $70 \%$ dos entrevistados.

A ferramenta de maior alcance e aplicação foi 'viagens e visitas a feiras', e apresentou o menor intervalo entre o aplicado e o desejado. 'História compartilhada' compareceu com maior frequência para desconhecimento da técnica, $50 \%$ dos entrevistados. 
Neste grupo, observa-se que as com maior aplicação são relacionadas a desenvolver o capital relacional das empresas, por meio de ações com foco na sociedade (de responsabilidade social) e interações com feiras e empresas do exterior.

Figura 5 - Métodos e técnicas avaliadas para Mecanismos de coordenaccão social para controle e coordenação social para os núcleos setoriais da ACIJS

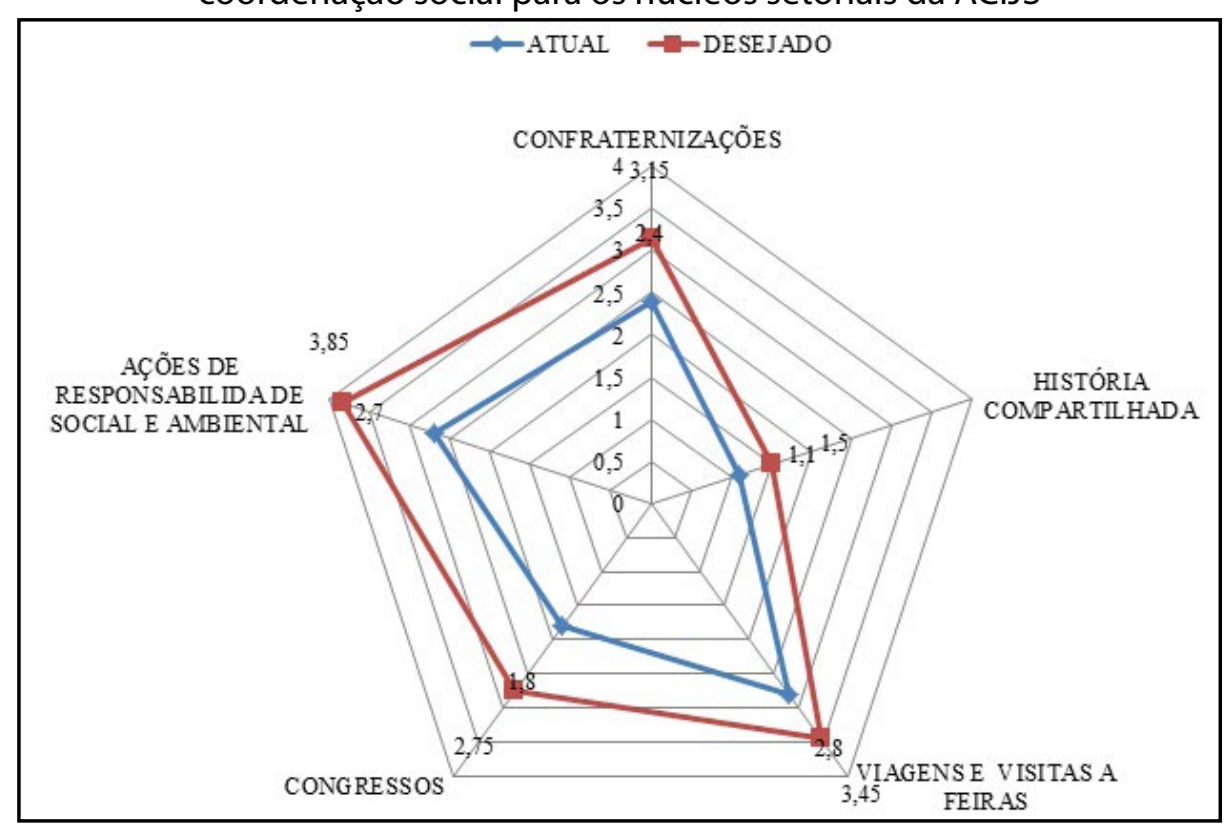

Fonte: Os autores (2013)

Mecanismos de coordenação para unidade e papéis de integração, 'páginas amarelas' foi frequente em $90 \%$ dos participantes para escala 'zero', no sentido de ignorar a ferramenta. A única organização a utilizar essa ferramenta classificou-a com "certo alcance e aplicação", escala "2". 'Planejamento estratégico envolvendo colaboradores' apresentou escala máxima de valor para 70\% dos entrevistados; na escala geral, atingiu "certo alcance de aplicação. 'Banco de competências individuais' de aplicação praticamente nula. No entanto, para os que utilizam, foi referendada sua importância.

Nos Mecanismos de coordenação para controle e planejamento, apresentados na Figura 6, 'narrativas' foi unanimidade no desconhecimento dos entrevistados. Outra técnica com frequência de $80 \%$ de desconhecimento e aplicação foi 'inteligência competitiva'.

A técnica mais aplicada foi 'elaboração de manuais para replicação de práticas', assim como foi a mais importante, considerada no intervalo de "uma grande aplicação" nas organizações avaliadas. Em seguida, foram o 'benchmarking interno e externo' e 'rotinas bem-sucedidas' quanto ao uso e, uma inversão de ordem nas posições para situações desejadas. Os dados estão representados graficamente na Figura 6.

Fica evidente que as três técnicas mais aplicadas têm influência direta no capital estrutural das empresas participantes. São exemplos a'explicitação do conhecimento em manuais', o'benchmarking interno e externo', que permitem a incorporação de novos ou processos produtivos melhorados, e a disseminação de rotinas bem-sucedidas. Assim, é possível afirmar que as empresas têm uma predisposição e capacidade importante de aprendizagem. 
Figura 6 - Métodos e técnicas avaliadas para Mecanismos de Coordenação Social para controle e coordenação social para os núcleos setoriais da AĆlJS

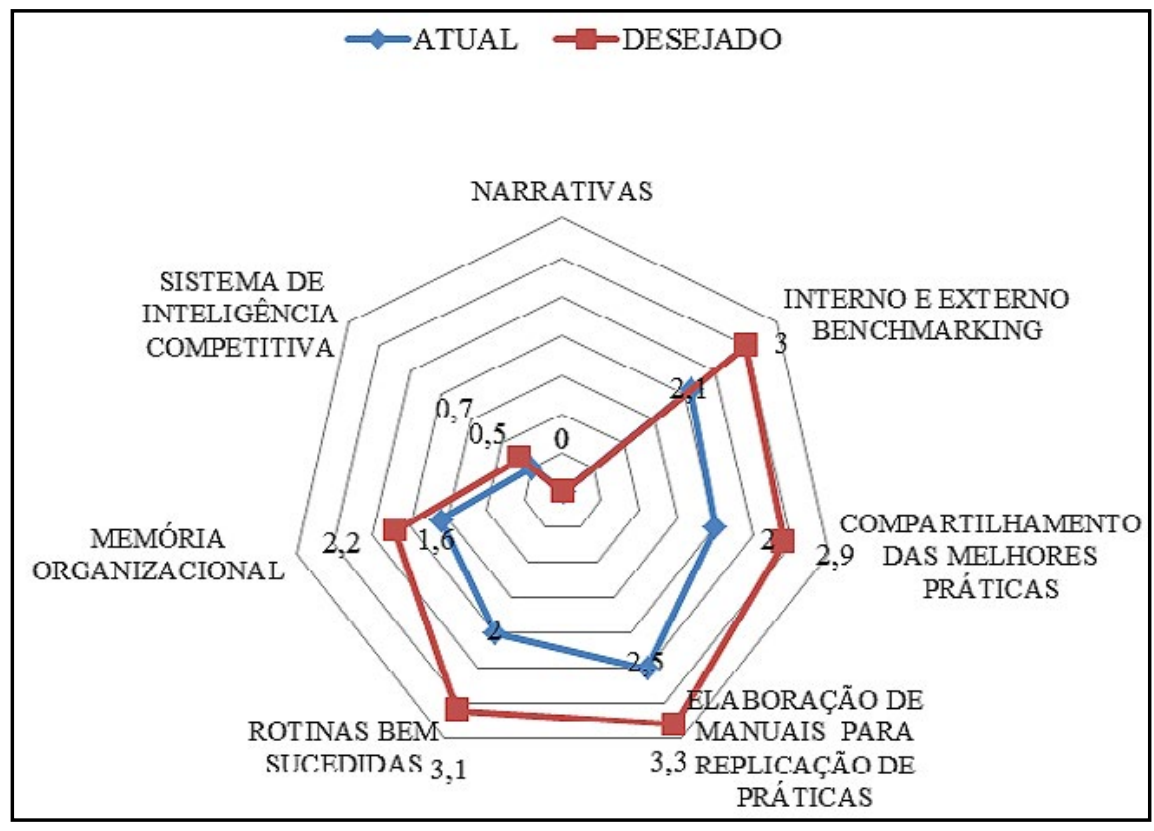

Fonte: Os autores (2013)

Em sistemas de incentivos para Mecanismos de coordenação, 'dia de inovação' obteve a menor aplicabilidade, frequência de $70 \%$ de desconhecimento. Dos integrantes que aplicavam essa técnica, foram unânimes em referendarem grau máximo de aplicabilidade para sistemas de incentivo. 'Estímulos para inovação' e 'educação continuada', ambas com mesmo índice, formaram o segundo grupo dos métodos de menor uso pelas organizações. Diferenciaram-se pelo escore de situação desejada, em que 'estímulo para inovação' esteve como terceira ferramenta para importância na aplicação, com 2.95 pontos, e a maior amplitude de pontos entre o executado e aplicação desejada.

O 'reembolso de despesas' foi configurado como o maior incentivador na prática da inovação e o mais desejado ( $90 \%$ nas duas escalas máximas de aplicação desejada). A segunda ferramenta mais utilizada e aplicação desejada foi 'treinamento, cursos, seminários, palestras, workshops'.

Os treinamentos para aprimorar a produtividade, assim como os estímulos para inovação são práticas de GC que influenciam diretamente no capital humano da empresa, conforme apresentado na Quadro 1. O fato é altamente relevante, pois permite criar círculos inovadores positivos (aprendizagem, inovação, recompensa), considerando que a inovação é a principal ferramenta de competitividade no mundo atual.

Figura 7 - Métodos e técnicas avaliadas para Mecanismos de Coordenação Social para incentivo à inovação para os núcleos setoriais da ACIJS

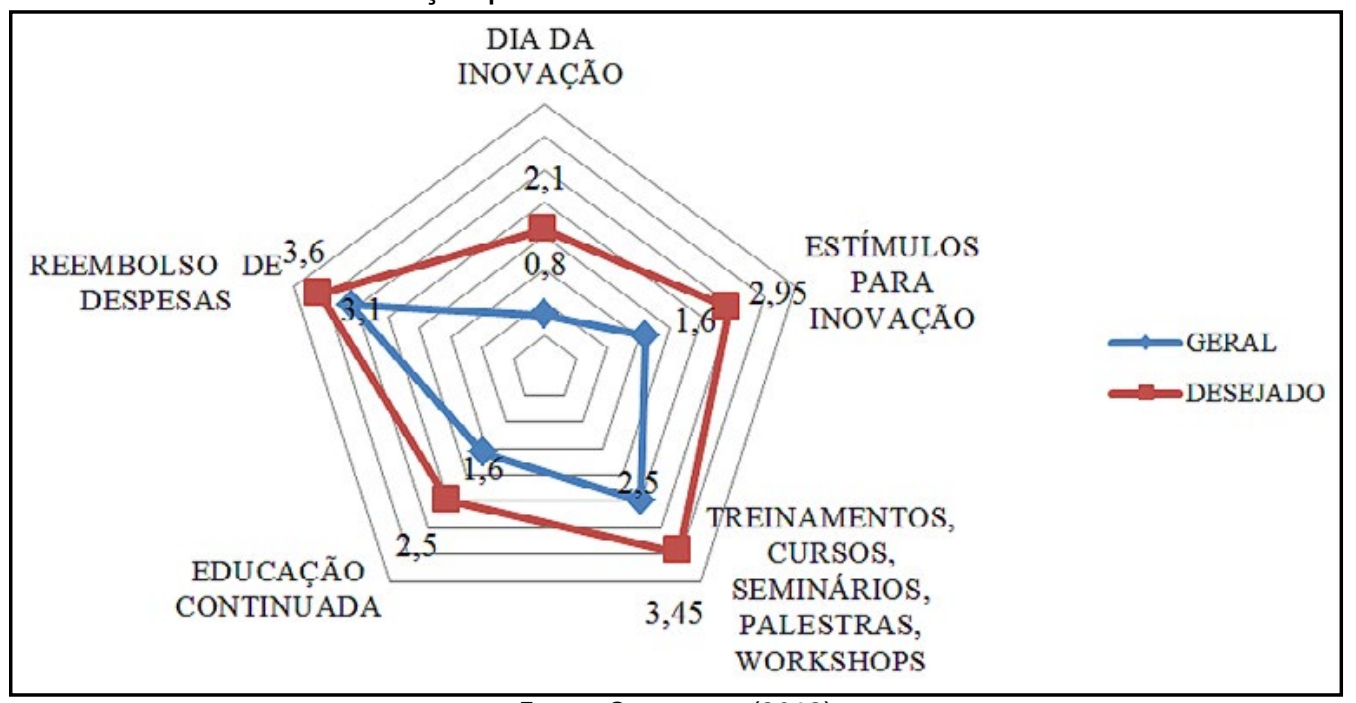

Fonte: Os autores (2013) 
Mecanismos de coordenação para sistemas de informação'espaços eletrônicos (portais, e-mails, chats, intranet e extranet)' foi o mais utilizado e mais valorado para aplicação na empresa - $90 \%$ de frequência na escala de situação desejada de aplicação muito grande. 'Gestão eletrônica de documentos' foi elencada como a segunda no atual alcance da ferramenta dentro da organização, bem como na aplicação desejada.

'Base de dados compartilhados on-line' e 'espaço eletrônico: e-learning" foram de baixo uso, posicionaram-se na primeira e terceira posição quanto a aplicação e com alta frequência para a falta de informação sobre a técnica, respectivamente. 'Gestão de conteúdo' também foi elencada no grupo dos métodos com grau de desconhecimento. No entanto, apresentou escala máxima para situação desejada de aplicabilidade para as organizações que aplicam a ferramenta.

A gestão eletrônica dos documentos e os espaços virtuais colaborativos (e-mail, internet, entre outros) representam práticas de GC que aprimoram diretamente o capital estrutural das organizações, por intermédio de uma melhor explicitação e compartilhamento do conhecimento.

Figura 8 - Métodos e técnicas avaliadas para Mecanismos de coordenação social para sistemas de informação para os núcleos setoriais da ACIJS

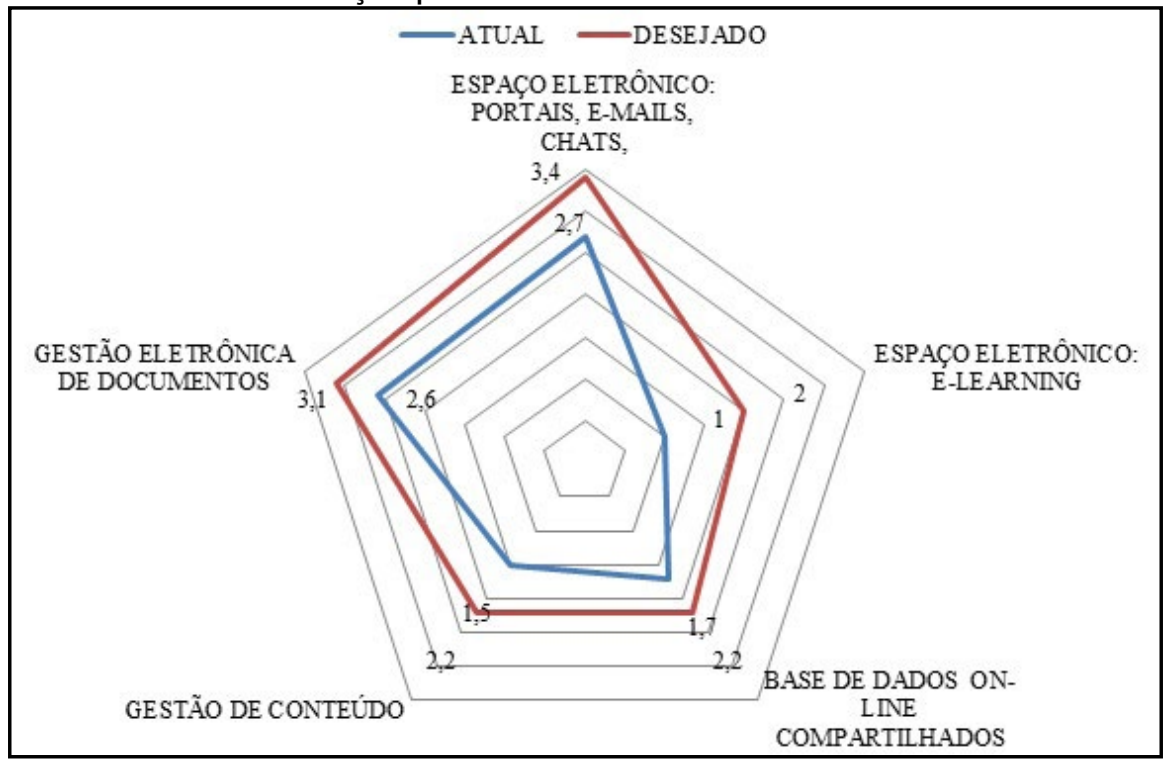

Fonte: Os autores (2013)

Foi relatado que os métodos e técnicas de GC que são utilizados, pelas empresas participantes da pesquisa, contribuem para a gestão do seu capital humano, estrutural e relacional. O Quadro 3 associa os métodos mais utilizados com a dimensão do capital intelectual suportada por cada ferramenta, com base nos resultados da pesquisa.

Quadro 3 - Métodos de GC mais utilizados que suportam a gestão do Capital Intelectual

\begin{tabular}{|c|c|c|}
\hline CAPITAL HUMANO & CAPITAL ESTRUTURAL & CAPITAL RELACIONAL \\
\hline \multicolumn{3}{|c|}{ Conexões interpessoais } \\
\hline 3 - Ajuda semelhante & & \\
\hline \multicolumn{3}{|c|}{ Memória Organizacional } \\
\hline \multicolumn{3}{|l|}{7 - Reuniões Informais } \\
\hline \multicolumn{3}{|l|}{9 - Grupos para solução } \\
\hline \multicolumn{3}{|c|}{ Mecanismo de Coordenação Social - Controle e Coordenação } \\
\hline & & 3 -Visitas técnicas \\
\hline \multicolumn{3}{|c|}{ Mecanismo de Coordenação Social - Controle e Coordenação } \\
\hline & & 5 -Viagens, visitas a feiras \\
\hline & & $\begin{array}{l}7 \text { - Ações de responsabilidade social e } \\
\text { ambiental }\end{array}$ \\
\hline \multicolumn{3}{|c|}{ Mecanismo de Coordenação Social - Controle e Planejamento } \\
\hline & $\begin{array}{l}5 \text { - Elaboração de manuais para } \\
\text { replicar boas práticas }\end{array}$ & \\
\hline
\end{tabular}




\begin{tabular}{|l|l|l|}
\hline & 6- Rotinas bem-sucedidas & \\
\hline & 9 - Benchmarking interno e externo & \\
\hline \multicolumn{3}{|c|}{ Sistema de Incentivo } \\
\hline 16 - Estímulo à inovação & & \\
\hline $\begin{array}{l}17 \text { - Treinamentos, cursos, palestras } \\
\text { e workshops }\end{array}$ & & \\
\hline 19 - Reembolso de despesas & \multicolumn{1}{|c|}{ Sistema de Informação } \\
\hline \multicolumn{3}{|c|}{} \\
\hline & 11 - Espaço eletrônico, e-learning & \\
\hline & $\begin{array}{l}13-\text { Gestão eletrônica de docu- } \\
\text { mentos }\end{array}$ & \\
\hline
\end{tabular}

Fonte: Os autores (2013)

\section{CONCLUSÕES}

Gerenciar adequadamente o conhecimento tem uma influência direta nos resultados da organização, nas dimensões de desempenho e da aprendizagem (APO, 2009). O gerenciamento é colocado em prática por meio de métodos e técnicas de GC que atuam de forma combinada. $O$ estudo proposto apresenta conclusões indicando caminhos fundamentados no framework de GC da APO (2010) por intermédio dos escores indicados pelos entrevistados e, posteriormente, quantificados e analisados.

Dentre 39 métodos e técnicas propostos como instrumentos para GC, somente dois foram avaliados, pelos entrevistados, como tendo realmente um grande alcance dentro da organização. Foram eles: 'reuniões informais' e 'reembolso de despesas'. Foram elencados como uma situação desejada para grande aplicação em suas empresas vinte métodos e técnicas.

Os métodos para atividade de conexão entre pessoas obtiveram pouco alcance na situação real das organizações, embora haja uma percepção da grande aplicação da 'técnica ajuda semelhante'.

Em relação aos mecanismos de memória organizacional, de dez propostas apresentadas, somente duas apresentaram certo alcance na GC ('reuniões informais' e 'grupos para soluções'). São identificados cinco métodos de grande aplicação na GC para as empresas arroladas, foram eles: 'foi certo ou foi errado', 'revisão pós-ação', 'revisão retrospectiva', 'grupos para solução' e 'publicações técnicas e operacionais'.

Para mecanismos de controle e coordenação social, somente 'reuniões informais' teve alcance grande como métodos de GC e foi indicado como uma situação de grande aplicação, apesar do desconhecimento de quatro métodos. Além dessa, foram elencadas, com grande aplicabilidade, 'confraternizações', 'viagens e visitas a feiras', e 'ações de responsabilidade social e ambiental'. Nos mecanismos de coordenação para controle e planejamento, não houve alcance para métodos utilizados, mas foram referendadas como grande aplicação 'memória organizacional', 'benchmarking interno e externo' e 'rotinas bem- sucedidas'.

O 'reembolso de despesas' foi técnica que obteve grande alcance como sistema de incentivo. Somou-se a esse método, indicado como promissores os 'treinamentos, cursos, seminários, palestras e workshop'. Para mecanismos de integração, destacou-se a aplicação do papel integrador na GC do 'planejamento estratégico envolvendo colaboradores'. Em mecanismos de coordenação para sistema de informação, não houve grande alcance para os métodos e técnicas avaliados no trabalho, mas foram destacados dois com grande aplicabilidade: 'portais, e-mail, chats, intranet e extranet' e 'gestão eletrônica de documentos'.

A recomendação da APO (2010) é que as técnicas de GC suportem adequadamente os processos de identificação, criação, aplicação, estoque e disseminação do conhecimento, criando valor com base em uma GC intencional, sistêmica e fortemente associada às estratégias organizacionais. Assim, o presente estudo revela que 'atividades de ajuda semelhante', 'revisão retrospectiva', 'visita técnica entre empresas', 'ações de responsabilidade sócio ambiental','elaboração de manuais para replicação de práticas' e 'gestão eletrônica de documentos' são métodos e técnicas de GC com diferenças entre a situação real de implementação e valorizadas para uma aplicação desejada. As diferenças devem ser analisadas e, se for o caso, resolvidas mediante projetos de implementação/melhoria. 


\title{
KNOWLEDGE MANAGEMENT METHODS AND TECHNIQUES APPLIED TO IMPROVE INTELLECTUAL CAPITAL MANAGEMENT IN A BUSINESS ASSOCIATION: "ACIJS"
}

\begin{abstract}
The importance of knowledge, demonstrated over the centuries, is fundamental to the economic, social and environmental performance of any organization. Improving the identification, creation, storing, dissemination and application of knowledge is essential to develop and improve organizational processes towards sustainable development, where Intellectual Capital is essential. The objective of this research was to characterize and analyze the methods and techniques of Knowledge Management in two nuclei of the Commercial and Industrial Association of Jaraguá do Sul (ACIJS). The sample included ten companies accounting for $25 \%$ of the active participants of the sector nuclei: the Corporate Sustainability Management Nucleus and Management and Quality Nucleus. Questionnaires created by the Asian Productive organization (APO- 2010) were applied to representatives of the two sectors. Responses characterized the current situation and the desired situation, graded in a scale from zero to four. They showed that a significant number of methods and techniques for knowledge management have already been implemented and that, when properly applied, these techniques and methods account for an adequate management of intelectual capital. The main methods and techniques applied were peer assistance, technical visits to companies, tours and attendance to fairs, elabboration of a guide to replicate practices, refund of expenses, eletronic communcation: e-mail - chats. Two tools were evaluated as having great importance within the organization. They were: informal meetings and refund of expenses. Twenty tools and techniques were listed as a desired situation for wide application in companies.
\end{abstract}

Keywords: Knowledge Management. Tools. Techniques. Intellectual capital

\section{REFERÊNCIAS}

ASIAN PRODUCTIVITY ORGANIZATION (APO). Knoweledge Management: Case Studies for Small and Medium Enterprises. Tokyo: APO, [2009].

ASIAN PRODUCTIVITY ORGANIZATION (APO). APO Knowledgre Management Tools and Techniques Manual. Tokyo: APO, [2010].

BONTIS, N. Assessing knowledge assets: a review of the models used to measure intellectual capital. International Journal of Management Reviews, Malden, MA, v. 3, issue 1, p. 41-60, 2001.

BUENO, E.; SALMADOR, M. P.; RODRÍGUEZ, O. Internal logic of intelectual capital: a biological approach. Journal of Intellectual Capital, Teleos, UK, v. 7, n. 3, p. 394-405, 2006.

BUKH, P. N.; LARSEN, H. T.; MOURITSEN, J. Constructing intellectual capital statements. Scandinavian Journal of Management, Gothenburg, Sweden, n. 17, p. 87-108, 2001.

CALVO, Leandro Cañibano et al. Guidelines for managing and reporting on intangibles: intellectual capital report. [2002]. Disponível em: <http://www.uam.es/personal_pdi/economicas/ palomas/DIRECTRICES\%20MERITUM\%20-\%20INGLES.pdf>. Acesso em: 9 jul. 2014.

COMITÉ EUROPEÉN DE NORMALISATION (CEN). European Guide to good Practice in Knowledge Management: Part 1 - 5. Brussels: European Committee for Standardization, 2004.

DALKIR, K. Knowledge management in theory and practice. Oxford: Elsevier ButterworthHeinemann, 2005.

DRUCKER, P. Management challenges for the twenty-first century. Oxon, UK: Routledge, 1999.

DRUCKER, P. F. Inovação e Espírito Empreendedor (entrepreneurship): prática e princípios. São Paulo: Pioneira, 2001.

FERENHOF, H. A.; BIALECKI, M. Z.; SELIG, P. M. Análise das Dimensões do Capital Intelectual: uma revisão de literatura. In: International Congress Of Kowledge And Inovation (ciKi), 3., 2013, Porto Alegre. Anais... Porto Alegre: Pontifícia Universidade Católica; Universidade Federal de Santa Catarina, 2013. 
GHANI., S. R. Knowledge Management: Tools and Techniques. Journal of Library \& Information Technology, v. 29, n. 6, p. 33-38, 2009.

GIULIANI, M.; MARASCA, S. Construction and valuation of intellectual capital: a case study. Journal of Intellectual Capital, Teleos, UK, v. 12, n. 3, p. 377-391, 2011.

HEISIG, P. Harmonisation of knowledge management - comparing $160 \mathrm{KM}$ frameworks around the globe. Journal of knowledge management, London, v. 13, n. 4, p. 4-31, 2009.

IDea. Knowledge Management Tools and Techniques: improvement and development agency for local government helping you access the right knowledge at the right time. [2008]. Disponível em: <www.idea.gov.uk/km>. Acesso em: 28 set. 2013.

KOMNENIC, B.; POKRAJCIC, D. Intellectual capital and corporate performance of MNCs in Serbia. Journal of Intellectual Capital, Teleos, UK, v. 13, n. 1, p. 106-119, 2012.

NAHAPIET, J.; GHOSHAL, S. Social capital, intellectual capital, and the organizational advantage. Academy of Management Review, v. 23, n. 2, p. 242-266, 1998.

NORTH, K.; RIVAS, R. Gestión del Conocimiento: Una guía práctica hacia la empresa inteligente. Buenos Aires: LibrosEnRed, 2010.

PORTER, M. E.; KRAMMER, M. R. The Big Idea: Creating Shared Value - How to re-invent capitalism - and unleash a wave of innovation and growth. [2011]. Disponível em: <http://www.hks.harvard. edu/m-rcbg/fellows/N_Lovegrove_Study_Group/Session_1/Michael_Porter_Creating_Shared_ Value.pdf >. Acesso em: 3 jul. 2014.

RAMALINGAM, B. Tools for Knowledge and Learning: A Guide for Development and Humanitarian Organisations. London: Research and Policy in Development Programme, 2006.

RODRIGUEZ, Martius; STOECKICHT, Ingrid Paola. Gestão Estratégica do Capital Intelectual voltada para a Inovação. In: MUNDIM, Ana Paula; RICARDO, Eleonora Jorge (Org.). Educação Corporativa: Fundamentos e Práticas. Rio de Janeiro: Qualitymark, 2004. p. 3-26.

SENGE, P. M. The fifth discipline: The art \& practice of the learning organization. São Paulo: Best Seller, 2000.

SERVIN, G. ABC of Knowledge Management. NHS National Library for Health. [2005]. Disponível em: <http://www.library.nhs.uk/knowledgemanagement>. Acesso em: 3 jul. 2014.

TSENG, K. et al. Mediation of strategy on intellectual capital and performance. Management Decision, Bengley, UK, v. 51, n. 7, p. 1488-1509, 2013.

YOUNG, R. Knowledge Management Tools and Techniques Manual. Tokyo: Asian Productivity Organization, 2010. 